\title{
Membranotropic Action of Pharmacological Agents
}

\author{
A. I. Vislobokov, Yu. D. Ignatov, and K. N. Melnikov \\ Valdman Institute of Pharmacology, Pavlov State University, St. Petersburg; \\ e-mail:Vislobokov@spmu.rssi.ru
}

DOI: $10.1134 / \mathrm{S} 1990747809030507$

Local anaesthetics, anti-arrythmic, analgesic, and psychotropic agents exert diverse membranotropic effects on a nerve cell. They increase (activate) or decrease (inhibit) ionic currents through voltage-gated sodium, calcium, and potassium channels; change the membrane surface charge in the vicinity of the ion channels; inactivate the channels or counteract their inactivation. Interactions of the pharmacological agents with channel gating structures result in alteration of the activation or inactivation kinetics. Membranotropic effects cause changes in membrane resting potential, synaptic potentials, and intercellular communications. Understanding the functioning mechanisms of neurons and possible ways of regulating their state pharmacologically makes the base of the concept of the cytopharmacological management of the cell functional state. 